\title{
The Construction of an Integral Formula for Computing Cylindrical and Non-Cylindrical Flow in the Region Bounded by Two Coaxial Cylinders of Varying Radii
}

\author{
Vasos Pavlika
}

\begin{abstract}
In this paper an integral formula will be derived that will compute the velocity of a non-cylindrical flow variation in swan neck ducts with prescribed flow characteristics. The flow can be characterized by the total head, $H(\psi)$ a function of only the stream function, $\psi(x, r)$ and by the function $C(\psi)$ which is a function of the azimuthal (circumferential) component of velocity vector, $u$ in cylindrical polar coordinates $(x, r, \theta)$. The solution to the problem is given in terms of an integral formula based on Green's first identity.
\end{abstract}

Index Terms-Stokes stream function, cylindrical flow, non-cylindrical flow, singular integral formula.

\section{INTRODUCTION}

This is the second paper in a series of two on cylindrical and non-cylindrical flow in the region bounded by two coaxial cylinders of varying radii. The first paper Pavlika [1] investigates all the possible cylindrical flow variations that may exist by considering the separation constant occurring in the differential equation that arises naturally when considering the flow in the region bounded by the coaxial cylinders. If coded and verified and such that software and numerical results were to be created and verified this paper would be lead to a breakthrough into a phenomenon that has never been observed, similar type investigations only being carried out by Taylor [2] but in which a closed form analytical solution has not been given in the literature. No such integral which eliminates singularities in the integrands is reported in the literature. The well known equation for the stream function $\psi(x, r)$, of a rotating fluid has been examined with a view of choosing a new dependent variable in such a way that the differential operator becomes the axisymmetric form of the Laplace operator (see for example Arfken [3]), this is advantageous since this makes the setting up of an equivalent integral formula based on Green's first identity, Roach [4], possible since a singular solution to the adjoint equation can be obtained. A suitable choice turns out to be the axial component of the velocity vector, $u_{x}$, and for a class of flows including solid body rotation, $u_{x}$ is shown to satisfy the Helmholtz equation subject to an oblique derivative boundary condition. Stoke's stream function $\psi(x$, $r$ ) for steady axisymmetric swirling flow of an incompressible inviscid fluid satisfies the equation:

Manuscript received June 21, 2013; revised September 25, 2013.

Vasos Pavlika is with SOAS, University of London, UK (e-mail: vp4@soas.ac.uk).

$$
\frac{\partial^{2} \psi}{\partial x^{2}}+\frac{\partial^{2} \psi}{\partial r^{2}}-\frac{1}{r} \frac{\partial \psi}{\partial r}=r^{2} \frac{d H}{d \psi}-C \frac{d C}{d \psi}
$$

where $H(\psi)$ is the total head and $C(\psi)=r u_{\vartheta}$, with the subscript $\theta$ denoting the circumferential component in cylindrical polar coordinates. The formulation would have application in the so-called "swan neck" duct connecting the compressor (or turbines) in a multishaft gas turbine. As shown in Pavlika [1] after using the Euler-Poisson-Darboux equation (see Weinstein [5) it can be shown that axisymmetric form of the Helmholtz equation is satisfied by the axial component of the velocity. So commencing with the three dimensional Helmholtz equation:

$$
\nabla^{2} u+A_{C} u=0
$$

which is self adjoint so that

$$
\nabla^{2} v+A_{C} v=0
$$

To progress to a solution it turns out to be more convenient to work in spherical polar coordinates $(R, \vartheta, \phi)$ to obtain the centro-symmetric fundamental solution $V(R)$ so that

$$
\begin{gathered}
\frac{1}{R^{2}} \frac{\partial}{\partial R}\left(R^{2} \frac{\partial v}{\partial R}\right)+\frac{1}{R^{2} \sin \vartheta} \frac{\partial}{\partial \vartheta}\left(\sin \vartheta \frac{\partial v}{\partial \vartheta}\right) \\
+\frac{1}{R^{2} \sin ^{2} \vartheta} \frac{\partial^{2} v}{\partial \phi^{2}}+A_{C} v=0
\end{gathered}
$$

With $v$ purely a function of the radius $R$, then $v=f(R) \Rightarrow$

$$
\frac{1}{R^{2}} \frac{d}{d R}\left(R^{2} \frac{d v}{d R}\right)+A_{C} v=0
$$

which can be written as

$$
\frac{d^{2}}{d R^{2}}(R v)=-A_{C} R v
$$

and has the general solution

$$
v(R)=E \cdot \frac{e^{-i \sqrt{A_{C}} R}}{R}+D \cdot \frac{e^{i \sqrt{A_{C}} R}}{R}
$$




$$
\frac{1}{2} \frac{d C^{2}}{d \psi}=A \psi+B
$$

where $E$ and $D$ are constants. Defining the region $\Gamma$ as the interior of the closed surface $S$, comprising the duct outer and inner walls and the upstream and downstream planes, then the solution of the three dimensional Helmholtz equation at a point $P$ in $\Gamma$ can be expressed as Green's formula as

$$
4 \pi u_{P}=\iint_{S}\left(u \frac{\partial v}{\partial n}-v \frac{\partial u}{\partial n}\right) d s
$$

where $v$ satisfies Helmholtz equation in $\Gamma$ except at $\mathrm{P}$ where it diverges like $1 /\left|\underline{x}-\underline{x}_{P}\right|$. A suitable fundamental solution can be obtained from the real part of equation (2)

$$
v(R)=\frac{\cos \left(\sqrt{A_{C}}\left|x-x_{P}\right|\right)}{\left|\underline{x}-\underline{x_{P}}\right|}=\frac{\cos \left(\sqrt{A_{C}} R\right)}{R},
$$

where $R=\left|\underline{x}-\underline{x}_{P}\right|$. In cylindrical polar coordinates $(y, \alpha, x)$, with $\mathrm{P}$ in the plane $\alpha=0$

$$
\left|\underline{x}-\underline{x}_{P}\right|=\left(y_{p}^{2}+y^{2}+2 y_{p} y \cos \alpha+\left(x-x_{p}\right)^{2}\right)^{1 / 2}
$$

so that $\mathrm{v}$ and $\partial v / \partial n$ depend on $\alpha$ but $U_{x}$ being an axisymmetric solution of equation (1) does not, so that formula (4) gives

$$
\begin{aligned}
& 4 \pi\left(U_{x}\right)_{P}= \\
& \oint_{\Sigma}\left\{U_{x} V^{\prime}\left(x, y, \underline{x}_{P}\right)-\frac{\partial}{\partial n}\left(U_{x}\right) V\left(x, y, \underline{x}_{P}\right)\right\} y d s
\end{aligned}
$$

where

$$
V\left(x, y, \underline{x}_{P}\right)=\int_{0}^{2 \pi} v\left(\left|\underline{x}-\underline{x}_{P}\right|\right) d \alpha
$$

and

$$
V^{\prime}\left(x, y, \underline{x}_{P}\right)=\int_{0}^{2 \pi} \frac{\partial}{\partial n} v\left(\left|\underline{x}-\underline{x}_{P}\right|\right) d \alpha
$$

The contour integral in formula (5) is taken around $\Sigma$ the intersection of the plane $\alpha=0$ with $\mathrm{S}$ and the normal direction is always into $\Gamma$ where the normals are drawn into $\Gamma$ only on the inner cylinder and downstream plane. Boundary conditions can now be used to replace $\frac{\partial}{\partial n}\left(U_{x}\right)$ on the inner and outer duct walls, while on the upstream and downstream planes, from the assumption of non-cylindrical flow:

$$
\frac{\partial}{\partial n}\left(U_{x}\right)= \pm \frac{\partial}{\partial x}\left(U_{x}\right) \neq 0
$$

where the \pm indicates a sign convention. A suitable integration by parts of the integral contribution of the term

$$
y \tan \beta \frac{\partial}{\partial s}\left(U_{x}\right)
$$

along the inner and outer cylinders then yields a formula for $U_{x}$ in $\Gamma$ in terms of its values on $\Sigma$. The usual limit of this formula as $\mathrm{P}$ tends to any point on $\Sigma$ finally provides a singular integral equation (of the second kind) for $u_{x}$. The functions $K$ and $K^{\prime}$ can be expanded in terms of the first and second complete elliptic integrals of modulus $k$ where

$$
k^{2}=\frac{4 y y_{p}}{\left(y+y_{p}\right)^{2}+\left(x-x_{p}\right)^{2}}
$$

The boundary condition is used only to substitute for $y \frac{\partial}{\partial n}\left(U_{x}\right)$

$$
\begin{aligned}
& \Rightarrow y \frac{\partial}{\partial n}\left(U_{x}\right)=y \tan \beta \frac{\partial}{\partial s}\left(U_{x}\right)+ \\
& \cos \beta\left\{\frac{1}{2} R^{\prime \prime}(x) \cdot\left(U_{x}+\frac{2 b}{A_{C}}\right)+y^{2} \frac{d H}{d \psi}-\frac{d}{d \psi}\left(\frac{1}{2} C^{2}\right)\right\}
\end{aligned}
$$

where

$$
y \tan \beta=R^{\prime}(x) / 2
$$

\section{DERIVATION OF AN INTEGRAL FORMULA TO ALLOW FOR THE POSSIBILITY OF NON-CYLINDRICAL FLOW UPSTREAM AND DOWNSTREAM}

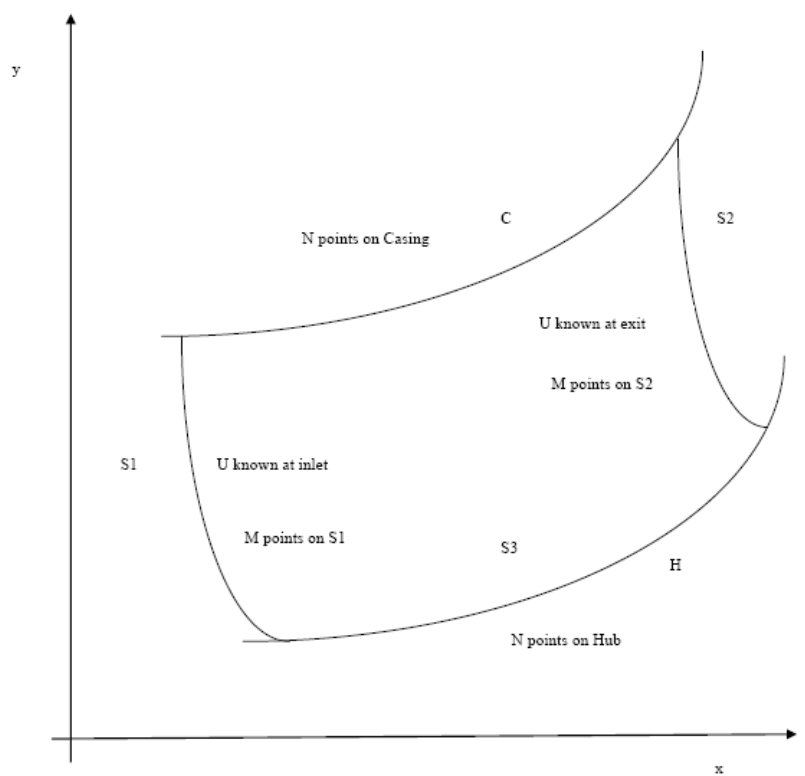

Fig. 1. Diagram showing the discs $S 1, S 2$ and $S 3$ in application to the integra formula.

Let there exist a flow of fluid along a duct of varying radii in the axial direction. The cylinder of the flow is bounded by 
the discs $S_{1}$ and $S_{2}$ and by a channel wall $S_{3}$ (as shown in Fig. 1). On the disc $S_{1}$ :

$$
\frac{\partial}{\partial n}=\frac{\partial}{\partial x_{q}}
$$

and

$$
d s_{q}=y_{q} d \alpha d y_{q}
$$

On the disc $S_{2}$ :

$$
\frac{\partial}{\partial n}=-\frac{\partial}{\partial x_{q}}
$$

and

$$
d s_{q}=y_{q} d \alpha d y_{q}
$$

On the channel wall $S_{3}$ :

$$
\begin{aligned}
& \frac{\partial}{\partial s}=\frac{\partial x_{q}}{\partial s_{q}} \frac{\partial}{\partial x_{q}}+\frac{\partial y_{q}}{\partial s_{q}} \frac{\partial}{\partial y_{q}} \\
& =\cos \beta_{q} \frac{\partial}{\partial x_{q}}+\sin \beta_{q} \frac{\partial}{\partial y_{q}}
\end{aligned}
$$

and

$$
\frac{\partial}{\partial n}=\frac{\partial x_{q}}{\partial n_{q}} \frac{\partial}{\partial x_{q}}+\frac{\partial y_{q}}{\partial n_{q}} \frac{\partial}{\partial y_{q}}
$$

on the outer wall:

$$
\frac{\partial}{\partial n}=\sin \beta_{q} \frac{\partial}{\partial x_{q}}-\cos \beta_{q} \frac{\partial}{\partial y_{q}}
$$

on the inner wall

$$
\frac{\partial}{\partial n}=-\sin \beta_{q} \frac{\partial}{\partial x_{q}}+\cos \beta_{q} \frac{\partial}{\partial y_{q}}
$$

Now $U_{x}$ satisfies Helmholtz equation thus also satisfies the integral formula

$$
\begin{gathered}
2 \pi\left(U_{x}\right)_{P}=\iint_{s_{1}-s_{2}}\left\{\begin{array}{c}
\left(U_{x}\right)_{q} \frac{\partial}{\partial x_{q}}\left(\frac{\cos \left(\sqrt{A_{C}} R\right)}{R}\right) \\
-\frac{\cos \left(\sqrt{A_{C}} R\right)}{R} \frac{\partial}{\partial x_{q}}\left(U_{x}\right)_{q}
\end{array}\right\} y_{q} d \alpha d y_{q} \\
-\iint_{H-C}\left\{\begin{array}{l}
\left(U_{x}\right)_{q}\left[\sin \beta_{q} \frac{\partial}{\partial x_{q}}\left(\frac{\cos \left(\sqrt{A_{C}} R\right)}{R}\right)\right. \\
\left.\cos \beta_{q} \frac{\partial}{\partial y_{q}}\left(\frac{\cos \left(\sqrt{A_{C}} R\right)}{R}\right)\right] \\
-\frac{\cos \left(\sqrt{A_{C}} R\right)}{R} \frac{\partial}{\partial n}\left(U_{x}\right)_{q}
\end{array}\right\} d s_{q}
\end{gathered}
$$

information is now required about the normal derivative of $U_{x}$. So that on

$$
S_{1}: \frac{\partial}{\partial x_{q}}\left(U_{x}\right)=\frac{\partial}{\partial x_{q}}\left(u_{x}-\frac{2 b}{A_{C}}\right)=0,
$$

for cylindrical flow and

$$
S_{1}: \frac{\partial}{\partial x_{q}}\left(U_{x}\right)=\frac{\partial}{\partial x_{q}}\left(u_{x}-\frac{2 b}{A_{C}}\right) \neq 0,
$$

for non-cylindrical flow

$$
S_{2}: \frac{\partial}{\partial x_{q}}\left(U_{x}\right)=-\frac{\partial}{\partial x_{q}}\left(u_{x}-\frac{2 b}{A_{C}}\right)=0,
$$

for cylindrical flow and

$$
S_{2}: \frac{\partial}{\partial x_{q}}\left(U_{x}\right)=-\frac{\partial}{\partial x_{q}}\left(u_{x}-\frac{2 b}{A_{C}}\right) \neq 0,
$$

for non-cylindrical flow on the channel walls

$$
\frac{\partial}{\partial n}\left(U_{x}\right)=\frac{\partial}{\partial n}\left(u_{x}-\frac{2 b}{A_{C}}\right)=\frac{\partial u_{x}}{\partial n}
$$

and as previously shown (on the inner wall)

$$
\frac{\partial}{\partial n}\left(U_{x}\right)=\frac{1}{y} \frac{\partial}{\partial s}\left(y u_{y}\right)-\frac{\cos \beta}{y}\left(A_{C} \psi+B_{C}-b y^{2}\right)
$$

Introducing

$$
u_{x}=u \cos \beta, u_{y}=u \sin \beta,
$$

$$
U_{x}=U \cos \beta=u \cos \beta-2 b / A_{C}
$$

so that $u=U+2 b(\sec \beta) / A_{C}$

and

$u_{y}=U \sin \beta+2 b(\tan \beta) / A_{C}$, so on the inner wall

$$
\begin{aligned}
& \frac{\partial}{\partial n}\left(U_{x}\right)=\frac{1}{y_{q}} \frac{\partial}{\partial s_{q}}\left[U_{q} y_{q} \sin \beta_{q}+2 b y_{q}\left(\tan \beta_{q}\right) / A_{C}\right. \\
& \left.-\cos \beta_{q}\left(A_{C} \psi+B_{C}-b y_{q}^{2}\right) / y_{q}\right]
\end{aligned}
$$

and on the outer wall

$$
\begin{aligned}
& \frac{\partial}{\partial n}\left(U_{x}\right)=-\frac{1}{y_{q}} \frac{\partial}{\partial s_{q}}\left[U_{q} y_{q} \sin \beta_{q}+\frac{2 b}{A_{C}} y_{q} \tan \beta_{q}\right. \\
& \left.+\cos \beta_{q}\left(A_{C} \psi+B_{C}-b y_{q}^{2}\right) / y_{q}\right]
\end{aligned}
$$

Hence

$$
\begin{aligned}
& 2 \pi \cos \beta_{p} U_{p}=\iint_{s_{1}-s_{2}}\left\{U_{q} \cos \beta_{q} \frac{\partial}{\partial x_{q}}\left(\frac{\cos \left(\sqrt{A_{C}} R\right)}{R}\right)\right\} y_{q} d \alpha d y_{q} \\
& +\iint_{s_{1}-s_{2}} \frac{\cos \left(\sqrt{A_{C}} R\right)}{R}\left(\begin{array}{l}
U_{q} \sin \beta_{q} \frac{\partial}{\partial x_{q}}\left(\beta_{q}\right) \\
-\cos \beta_{q} \frac{\partial U_{q}}{\partial x_{q}}
\end{array}\right) y_{q} d \alpha d y_{q}
\end{aligned}
$$


$-\iint_{H-C}\left\{\begin{array}{l}U_{q} \cos \beta_{q}\left[\sin \beta_{q} \frac{\partial}{\partial x_{q}}\left(\frac{\cos \left(\sqrt{A_{C}} R\right)}{R}\right)\right. \\ \left.-\cos \beta_{q} \frac{\partial}{\partial y_{q}}\left(\frac{\cos \left(\sqrt{A_{C}} R\right)}{R}\right)\right] \\ +\frac{\cos \left(\sqrt{A_{C}} R\right)}{R}\left[\frac{1}{y_{q}} \frac{\partial}{\partial s_{q}}\left[U_{q} y_{q} \sin \beta_{q}+\frac{2 b}{A_{C}} y_{q} \tan \beta_{q}\right]\right. \\ \left.-\frac{\cos \beta_{q}}{y_{q}}\left(A_{C} \psi+B_{C}-b y_{q}^{2}\right)\right]\end{array}\right\} y_{q} d \alpha d y_{q}$

III. DeRIVATIVE TERMS

If $R$ is the distance from any point $q$ on either surface of the annulus to fixed point $p$ then

$$
\begin{aligned}
& R^{2}=y_{p}^{2}+y_{q}^{2}-2 y_{p} y_{q} \cos \alpha+\left(x_{q}-x_{p}\right)^{2} \\
& \Rightarrow \frac{\partial}{\partial x_{q}}\left(\frac{\cos \left(\sqrt{A_{C}} R\right)}{R}\right)= \\
& -\frac{\left(x_{q}-x_{p}\right)}{R^{3}}\left(\cos \left(\sqrt{A_{C}} R\right)+\sqrt{A_{C}} R \sin \left(\sqrt{A_{C}} R\right)\right)
\end{aligned}
$$

and

$$
\begin{aligned}
& \frac{\partial}{\partial y_{q}}\left(\frac{\cos \left(\sqrt{A_{C}} R\right)}{R}\right)= \\
& -\frac{\left(y_{q}-y_{p} \cos \alpha\right)}{R^{3}}\left(\cos \left(\sqrt{A_{C}} R\right)+\sqrt{A_{C}} R \sin \left(\sqrt{A_{C}} R\right)\right)
\end{aligned}
$$

Substituting into equation (6) gives

$$
\begin{aligned}
& 2 \pi \cos \beta_{p} U_{p}=\int_{S_{1}-S_{2}}\left(y_{q} \cos \beta_{q}\left(x_{p}-x_{q}\right) I^{(1)}\right) U_{q} d y_{q} \\
& +\int_{S_{1}-S_{2}}\left\{U_{q} \sin \beta_{q} \frac{\partial}{\partial x_{q}}\left(\beta_{q}\right)-\cos \beta_{q} \frac{\partial U_{q}}{\partial x_{q}}\right) I^{(3)} y_{q} d y_{q} \\
& -\int_{H-C}\left\{\begin{array}{l}
y_{q} \cos \beta_{q}\left[\sin \beta_{q}\left(x_{p}-x_{q}\right)+\cos \beta_{q} y_{q}\right] I^{(1)} U_{q} \\
-y_{p} y_{q} \cos ^{2} \beta_{q} I^{(2)} U_{q}
\end{array}\right\} d s_{q} \\
& -\int_{H-C}\left\{\begin{array}{l}
\frac{\partial}{\partial s_{q}}\left(\left[U_{q} y_{q} \sin \beta_{q}+\frac{2 b}{A_{C}} y_{q} \tan \beta_{q}\right]\right. \\
\left.-\cos \beta_{q}\left(A_{C} \psi+B_{C}-b y_{q}^{2}\right)\right] I^{(3)}
\end{array}\right\} d s_{q}
\end{aligned}
$$

where

$$
\begin{aligned}
& I^{(1)}=\int_{0}^{2 \pi} \frac{\cos \left(\sqrt{A_{C}} R\right)+\sqrt{A_{C}} R \sin \left(\sqrt{A_{C}} R\right)}{R^{3}} d \alpha \\
& I^{(2)}=\int_{0}^{2 \pi} \frac{\cos \left(\sqrt{A_{C}} R\right)+\sqrt{A_{C}} R \sin \left(\sqrt{A_{C}} R\right)}{R^{3}} \cos \alpha d \alpha \\
& I^{(3)}=\int_{0}^{2 \pi} \frac{\cos \left(\sqrt{A_{C}} R\right)}{R} d \alpha
\end{aligned}
$$

Integrating by parts over $\mathrm{H}-\mathrm{C}$ gives:

$$
\begin{aligned}
& 2 \pi \cos \beta_{p} U_{p}=\int_{S_{1}-S_{2}}\left(y_{q} \cos \beta_{q}\left(x_{p}-x_{q}\right) I^{(1)}\right) U_{q} d y_{q} \\
& +\int_{S_{1}-S_{2}}\left(U_{q} \sin \beta_{q} \frac{\partial}{\partial x_{q}}\left(\beta_{q}\right)-\cos \beta_{q} \frac{\partial U_{q}}{\partial x_{q}}\right) I^{(3)} y_{q} d y_{q} \\
& -\int_{H-C}\left\{\begin{array}{l}
\left.y_{q} \cos \beta_{q}\left[\sin \beta_{q}\left(x_{p}-x_{q}\right)+\cos \beta_{q} y_{q}\right] I^{(1)} U_{q}\right) \\
-\cos \beta_{q}\left(A_{C} \psi+B_{C}-b y_{q}^{2}\right) I^{(3)} U_{q}
\end{array}\right\} d s_{q} \\
& -\left[I^{(3)}\left(y_{q} U_{q} \sin \beta_{q}+\frac{2 b}{A_{C}} y_{q} \tan \beta_{q}\right)\right]_{\text {farupstream }}^{\text {fardownstream }} \\
& +\int_{H-C}\left\{\left(y_{q} U_{q} \sin \beta_{q}+\frac{2 b}{A_{C}} y_{q} \tan \beta_{q}\right)_{d}^{d}\left(I_{q}^{(3)}\right)\right\} d s_{q}
\end{aligned}
$$

Hence an integral formula to cope with the possibility of non-cylindrical flow upstream and downstream is obtained:

$$
\begin{aligned}
& 2 \pi \cos \beta_{p} U_{p}=\int_{S_{1}-S_{2}}\left(y_{q} \cos \beta_{q}\left(x_{p}-x_{q}\right) I^{(1)}\right) U_{q} d y_{q} \\
& +\int_{S_{1}-S_{2}}\left(U_{q} \sin \beta_{q} \frac{\partial}{\partial x_{q}}\left(\beta_{q}\right)-\cos \beta_{q} \frac{\partial U_{q}}{\partial x_{q}}\right) I^{(3)} y_{q} d y_{q} \\
& -\int_{H-C}\left\{\begin{array}{l}
y_{q} \cos \beta_{q}\left[\sin \beta_{q}\left(x_{p}-x_{q}\right)+\cos \beta_{q} y_{q}\right] I^{(1)} U_{q} \\
-y_{p} y_{q} \cos ^{2} \beta_{q} I^{(2)} U_{q} \\
-\cos \beta_{q}\left(A_{C} \psi+B_{C}-b y_{q}^{2}\right) I^{(3)}
\end{array}\right\} d s_{q} \\
& -\left[I^{(3)}\left(y_{q} U_{q} \sin \beta_{q}+\frac{2 b}{A_{C}} y_{q} \tan \beta_{q}\right)\right]_{\text {farupstream }}^{\text {fardownstream }}
\end{aligned}
$$

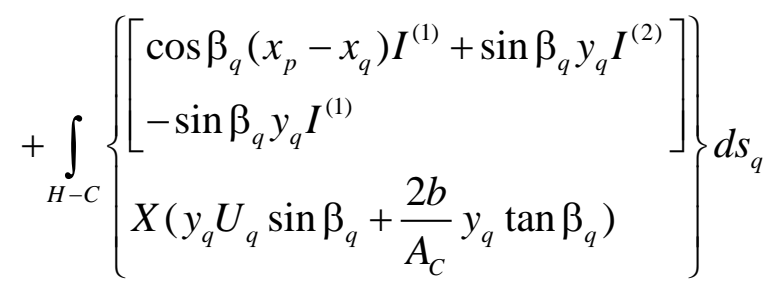

Considering the kernel of $I^{(3)}$ in the integral set (equation (7)) this can be written in the form:

$$
\begin{gathered}
\frac{\cos \left(\sqrt{A_{C}} R\right)}{R}=\sum_{n=0}^{\infty} \frac{(-1)^{n}}{(2 n) !} A_{C}^{n} R^{2 n-1} \\
R=\sqrt{2 y_{q} y_{p}}\left[\frac{y_{p}^{2}+y_{q}^{2}+\left(x_{P}-x_{q}\right)^{2}}{2 y_{p} y_{q}}-\cos \alpha\right]^{1 / 2} \\
=c\left(\kappa^{2}-\cos \alpha\right)^{1 / 2}
\end{gathered}
$$


where $c$ and $\kappa$ are defined by equation (9), at this point in the analysis the complete elliptic integrals of Legendre may be invoked to evaluate the integrals of the equation set (7) this would introduce the use of the following reduction formulae of the form:

$$
\begin{aligned}
& \int(a+b \cos \vartheta)^{m}(\alpha+\delta \cos \vartheta) d \vartheta \\
& =F(\vartheta)+\int(a+b \cos \vartheta)^{m-1}(A+B \cos \vartheta) d \vartheta
\end{aligned}
$$

This would not be a difficult exercise but an alternative method was preferred.

\section{THE NUMERICAL SOLUTION VIA THE INTEGRAL FORMULA}

In the integral formula (formula (8)), if far upstream and downstream (i.e. at $x= \pm \infty$ ) there is cylindrical flow (i.e. on the discs $S_{1}$ and $S_{2}$ ), then with slight manipulation, formula (8) can be written in the form

$$
U_{P}+\int_{H-C} K(p, q) U_{q} d s_{q}=R_{P}
$$

where

$$
K(p, q)=\left(y_{q}^{2} I^{(1)}-y_{p} y_{q} I^{(2)}\right) /\left(2 \pi \cos \beta_{p}\right)
$$

and defining

$$
\Pi_{P}=2 \pi \cos \beta_{p} .
$$

Then

$$
\begin{aligned}
& R_{P}=\int_{S_{1}-S_{2}} \frac{y_{q}\left(x_{p}-x_{q}\right) I^{(1)}}{\Pi_{P}} U_{q} d y_{q}+ \\
&\left.\left\{\begin{array}{l}
{\left[\frac{2 b}{A_{C}} y_{q} \tan \beta_{q}\left[\begin{array}{l}
\left(\cos \beta_{q}\left(x_{p}-x_{q}\right)-\sin \beta_{q} y_{q}\right) I^{(1)} \\
+\sin \beta_{q} y_{P} I^{(2)}
\end{array}\right]\right.}
\end{array}\right]\right\} \\
& \int_{H-C}\left\{\begin{array}{l}
\text { (os } \beta_{q}\left(A_{C} \psi+B_{C}-b y_{q}^{2}\right) I^{(3)} \\
+\cos
\end{array}\right] \\
& / \Pi_{P} d s_{q}
\end{aligned}
$$

taking a set of q positions $q_{1}, q_{2}, \ldots \ldots, q_{2 N}$ on the boundary positions on which $U$ is unknown, a suitable integration technique is used to represent each element of boundary contribution linearly in terms of the $q_{r}$ and $q_{r+1}$ values, that is replace the integral by a summation. Then let p occupy each of the q positions in turn (see Fig. 1 and Fig. 2), so that for $i=1,2, \ldots ., 2 N$

$$
U_{P_{i}}+\sum_{n=1}^{2 N} f\left(K\left(p_{i}, q_{n}\right) U_{q}\right) \Delta s_{q_{n}}=R_{p_{i}}
$$

For $i=1,2,3, \ldots, 2 N$. The function $\mathrm{f}$ of $\mathrm{KU}$ will of course depend on which numerical technique is being used. If the trapezoidal technique is being used then the equations become (for $i=1,2,3, \ldots, 2 N$ )

$$
U_{P_{i}}+\sum_{n=1}^{2 N} \frac{1}{2} K\left(p_{i}, q_{n}\right) U_{q_{n}}\left(s_{n+1}-s_{n-1}\right)=R_{p_{i}}
$$

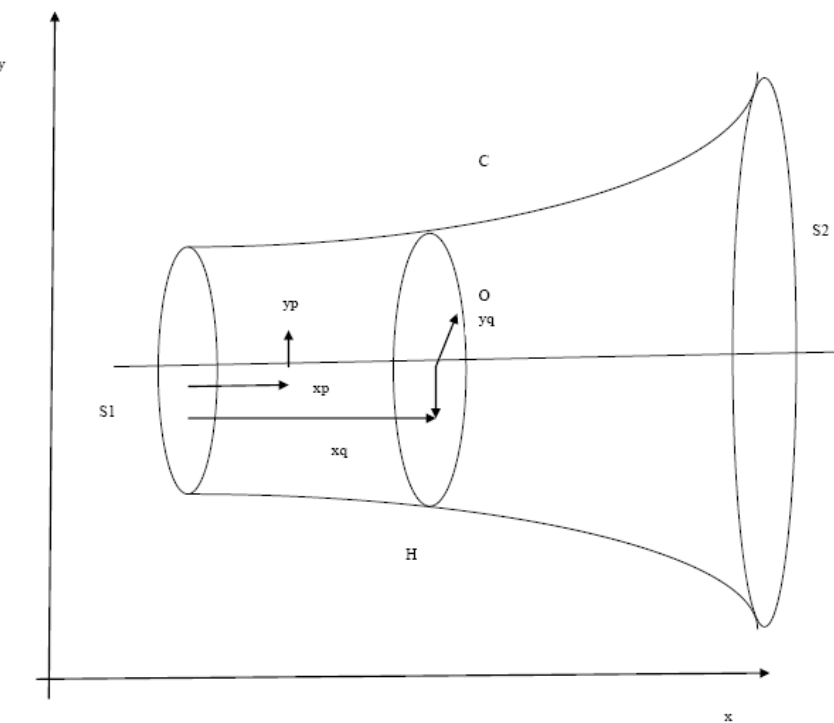

Fig. 2. Diagram showing the discs S1, S2 and S3 in application to the integral formula.

Note that $\mathrm{p}$ and $\mathrm{q}$ are no longer required so that

$$
U_{i}+\sum_{n=1}^{2 N} \frac{1}{2} K_{i, n} U_{n}\left(s_{n+1}-s_{n-1}\right)=R_{i}
$$

In matrix-vector form the equations become

$$
\begin{aligned}
& \left(1+\frac{1}{2} K_{1,1}\left(s_{2}-s_{0}\right), \quad \frac{1}{2} K_{1,2}\left(s_{3}-s_{1}\right),\right. \\
& \frac{1}{2} K_{2,1}\left(s_{2}-s_{0}\right), \quad 1+\frac{1}{2} K_{2,2}\left(s_{3}-s_{1}\right), \quad \frac{1}{2} K_{2,3}\left(s_{4}-s_{2}\right) .
\end{aligned}
$$$$
1+\frac{1}{2} K_{2 N, 2 N}\left(s_{2 N+1}-s_{2 N-1}\right)
$$$$
\left(\begin{array}{c}
U_{1} \\
U_{2} \\
\cdot \\
\cdot \\
\cdot \\
U_{2 N}
\end{array}\right)=\left(\begin{array}{c}
R_{1} \\
R_{2} \\
\cdot \\
\cdot \\
\cdot \\
R_{2 N}
\end{array}\right)
$$

i.e. $A \underline{U}=\underline{R}$. Equation (12) can now be solved by $\mathrm{LU}$ decomposition.

\section{SPECIAL CONSIDERATION WHEN THE INTEGRANDS BECOME SINGULAR}

Commencing with Commencing with the term

$$
\begin{aligned}
& R^{2}=y_{p}^{2}+y_{q}^{2}-2 y_{p} y_{q} \cos \alpha+\left(x_{q}-x_{p}\right)^{2} \\
& =\left(y_{p}-y_{q}\right)^{2}+2 y_{p} y_{q}-2 y_{p} y_{q} \cos \alpha+\left(x_{q}-x_{p}\right)^{2} \\
& =\left(y_{p}-y_{q}\right)^{2}+4 y_{p} y_{q} \sin ^{2}\left(\frac{\alpha}{2}\right)+\left(x_{q}-x_{p}\right)^{2}
\end{aligned}
$$

With all terms positive $R^{2}=0$, iff $y_{p}=y_{q} ; x_{p}=x_{q}$ and 
$\sin ^{2}\left(\frac{\alpha}{2}\right)=0$, so that $\alpha= \pm 2 n \pi, n=0,1,2, \ldots \ldots$. Writing the integral expressions (equations(s) (7)) in the form

$$
\begin{aligned}
& I^{(1)}=2 \int_{0}^{\pi} \mathfrak{R} e\left(\frac{\left(1+i \sqrt{A_{C}} R\right) e^{-i \sqrt{A_{C}} R}}{R^{3}}\right) d \alpha \\
& I^{(2)}=2 \int_{0}^{\pi} \Re e\left(\frac{\left(1+i \sqrt{A_{C}} R\right) e^{-i \sqrt{A_{C}} R}}{R^{3}}\right) \cos \alpha d \alpha \\
& I^{(3)}=2 \int_{0}^{\pi} \Re e\left(\frac{e^{-i \sqrt{A_{C}} R}}{R}\right) d \alpha
\end{aligned}
$$

With special attention required when $y_{p}=y_{q} ; x_{p}=x_{q}$; $\alpha=0$. Considering now the term

$$
K(p, q)=\left(y_{q}^{2} I^{(1)}-y_{p} y_{q} I^{(2)}\right) / \Pi_{p}
$$

appearing in equation (10), so that

$$
\begin{aligned}
& K(p, q)= \\
& \frac{2}{\Pi_{p}} \int_{0}^{\pi}\left(1+i \sqrt{A_{C}} R\right) e^{-i \sqrt{A_{C}} R}\left\{\frac{y_{q}^{2}}{R^{3}}-\frac{y_{p} y_{q} \cos \alpha}{R^{3}}\right\} d \alpha \\
& =\frac{2}{\Pi_{p}} \int_{0}^{\pi}\left(1+i \sqrt{A_{C}} R\right) e^{-i \sqrt{A_{C}} R} f\left(y_{p}, y_{q}, \alpha\right) d \alpha
\end{aligned}
$$

where

$$
\begin{aligned}
& f\left(y_{p}, y_{q}, \alpha\right)=\frac{y_{q}^{2}}{R^{3}}-\frac{y_{p} y_{q} \cos \alpha}{R^{3}}, \text { so that } \\
& K(p, q)=\frac{2}{\Pi_{p}}\left[\int_{0}^{\pi}\left(1+i \sqrt{A_{C}} R\right) e^{-i \sqrt{A_{c}} R} f\left(y_{p}, y_{q}, \alpha\right) d \alpha-\right. \\
& \left.\int_{0}^{\pi} f\left(y_{p}, y_{q}, \alpha\right) d \alpha \int_{0}^{\pi} f\left(y_{p}, y_{q}, \alpha\right) d \alpha\right] \\
& =\frac{2}{\Pi_{p}}\left[\int_{0}^{\pi}\left(1+i \sqrt{A_{C}} R-1\right) e^{-i \sqrt{A_{c}} R} f\left(y_{p}, y_{q}, \alpha\right) d \alpha\right. \\
& \left.+\int_{0}^{\pi} f\left(y_{p}, y_{q}, \alpha\right) d \alpha\right]
\end{aligned}
$$

Now

$$
\begin{aligned}
& \left(1+i \sqrt{A_{C}} R-1\right) e^{-i \sqrt{A_{c}} R}=\left(1+\frac{A_{C} R^{2}}{2}+\ldots . . .\right)-1 \\
& \Rightarrow K(p, q)=\frac{2}{\Pi_{p}}\left[\frac{A_{c} y_{q}}{2} \int_{0}^{2 \pi} \frac{\left(y_{q}-y_{p}\right)+y_{p}(1-\cos \alpha)}{\sqrt{\left(y_{q}-y_{p}\right)^{2}+4 y_{p} y_{q} \sin ^{2}\left(\frac{\alpha}{2}\right)+\left(x_{p}-x_{q}\right)^{2}}} d \alpha+\right. \\
& \left.+\int_{0}^{2 \pi} \frac{y_{q}\left(y_{q}-y_{p} \cos \alpha\right)}{\left(\left(y_{q}-y_{p}\right)^{2}+4 y_{p} y_{q} \sin ^{2}\left(\frac{\alpha}{2}\right)+\left(x_{p}-x_{q}\right)^{2}\right)^{3 / 2}} d \alpha\right]
\end{aligned}
$$

with $\alpha=0$ and $x_{p}-x_{q}=\delta x, y_{p}-y_{q}=\delta y$ the first term in equation (13) gives

$$
\begin{aligned}
& \lim _{\substack{\delta x \rightarrow 0 \\
\delta y \rightarrow 0}}\left(\frac{A_{C} y_{q}}{2} \int_{0}^{2 \pi} \frac{1}{\sqrt{1+\left(\frac{\delta x}{\delta y}\right)^{2}}} d \alpha\right) \\
& =A_{C} y_{q} \sin \beta \int_{0}^{\pi} d \alpha
\end{aligned}
$$

hence the integrand is finite in the limit as $\alpha=0$, $x_{p} \rightarrow x_{q}$, and $y_{p} \rightarrow y_{q}$ i.e. there is a removable singularity, with $x_{p}=x_{q}, y_{p}=y_{q}$ the second term in equation (13) gives:

$$
\frac{1}{8 y_{q}} \int_{-\pi}^{\pi} \frac{d \alpha}{\sin \left(\frac{\alpha}{2}\right)}=\frac{1}{8 y_{q}}\left[\log _{e}\left|\tan \frac{\alpha}{4}\right|\right]_{-\pi}^{\pi}=0
$$

limits of $-\pi$ to $\pi$ have been used since the integrand is even and integration through the singularity is performed. Therefore

$$
\begin{aligned}
& K(p, q)=\frac{1}{\Pi_{p}}\left[\left.\int_{0}^{\pi}\left(\frac{y_{q}}{R^{3}}-\frac{y_{q} y_{p} \cos \alpha}{R^{3}}\right) d \alpha\right|_{\substack{x_{p} \neq x_{q} \\
y_{p} \neq y_{q}}}+\right. \\
& \left.A_{C} \int_{0}^{\pi} \frac{\left(y_{q}-y_{p}\right)+y_{p}(1-\cos \alpha)}{\sqrt{\left(y_{q}-y_{p}\right)^{2}+4 y_{p} y_{q} \sin ^{2}\left(\frac{\alpha}{2}\right)+\left(x_{p}-x_{q}\right)^{2}}} d \alpha\right]
\end{aligned}
$$

which is now well behaved. The integrals can now be evaluated numerically, the notation $\left.\right|_{\substack{x_{p} \neq x_{q} \\ y_{p} \neq y_{q}}}$ denotes that in the integral the point where $\mathrm{p}$ and $\mathrm{q}$ coincide has been excluded since its integral contribution is zero. Now let's consider the term

$$
\begin{aligned}
& R_{P}^{\prime}=F\left(A_{C}, b, \beta_{q}\right) y_{q}\left(x_{q}-x_{p}\right) I^{(1)}-G\left(A_{C}, b, \beta_{q}\right) y_{q}^{2} I^{(1)} \\
& +G\left(A_{C}, b, \beta_{q}\right) y_{q}^{2} I^{(1)}+G\left(A_{C}, b, \beta_{q}\right) y_{q} y_{P} I^{(2)} \\
& +\left(H\left(A_{C}, b, \beta_{q}\right)-J\left(b, \beta_{q}\right)\right) I^{(3)}
\end{aligned}
$$

which occurs in the integral over $\mathrm{H}-\mathrm{C}$ in equation (11), where

$$
\begin{aligned}
& F\left(A_{C}, b, \beta_{q}\right)=2 b\left(\sin \beta_{q}\right) / A_{C} \Pi_{P} ; \\
& G\left(A_{C}, b, \beta_{q}\right)=2 b\left(\tan \beta_{q} \sin \beta_{q}\right) / A_{C} \Pi_{P} \\
& H\left(A_{C}, b, \beta_{q}\right)=\cos \beta_{q}\left(A_{C} \psi+B\right) / \Pi_{P} ; \\
& J\left(b, \beta_{q}\right)=b\left(\cos \beta_{q}\right) / \Pi_{P}
\end{aligned}
$$

dropping now the arguments in these temporarily introduced functions, considering the first three terms in expression (14) and denoting this integral as $R_{1}$ say then

$$
R_{1}=2\left\{\int_{0}^{\pi}\left[\frac{\left(1+i \sqrt{A_{C}} R\right) e^{-i \sqrt{A_{C}} R} .}{y_{q}\left(x_{P}-x_{q}\right)+G y_{q}\left(y_{P} \cos \alpha-y_{q}\right)} R^{3}\right] d \alpha\right\}
$$




$$
R_{1}=2\left\{\int_{0}^{\pi}\left(1+i \sqrt{A_{C}} R\right) e^{-i \sqrt{A_{C}} R} L\left(y_{P}, y_{q}, x_{P}, x_{q}, \alpha\right) d \alpha\right\}
$$

where $L\left(y_{P}, y_{q}, x_{P}, x_{q}, \alpha\right)$ follows from the integral expression (15), so that

$$
\begin{aligned}
& \left.R_{1}=2\left\{\int_{0}^{\pi}\left(1+i \sqrt{A_{C}} R\right) e^{-i \sqrt{A_{C}} R}-1\right) L d \alpha\right\}+2 \int_{0}^{\pi} L d \alpha \\
& =A_{C} \int_{0}^{\pi} R^{2} L\left(y_{P}, y_{q}, x_{P}, x_{q}, \alpha\right) d \alpha+2 \int_{0}^{\pi} L\left(y_{P}, y_{q}, x_{P}, x_{q}, \alpha\right) d \alpha
\end{aligned}
$$

Putting $\alpha=0, x_{P}-x_{q}=\delta x, y_{P}-y_{q}=\delta y$ the first term in equation (16) gives

$$
A_{C} G\left(A_{C}, b, \beta_{q}\right) \sin \beta \int_{0}^{\pi} d \alpha
$$

so the integrand is once again finite when $\alpha=0$, so that

$$
\begin{aligned}
& R_{1}=A_{C} \int_{0}^{\pi} R^{2} L\left(y_{P}, y_{q}, x_{P}, x_{q}, \alpha\right) d \alpha+ \\
& 2 \int_{0}^{\pi} L\left(y_{P}, y_{q}, x_{P}, x_{q}, \alpha\right) d \alpha
\end{aligned}
$$

With $x_{P}=x_{q}, y_{P}=y_{q}$ the second term in (17) gives

Hence

$$
\frac{G}{4 y_{q}}\left[\log _{e}\left|\tan \frac{\alpha}{4}\right|\right]_{-\pi}^{\pi}=0
$$

$$
\begin{aligned}
& R_{1}=A_{C} \int_{0}^{\pi} R^{2} L\left(y_{P}, y_{q}, x_{P}, x_{q}, \alpha\right) d \alpha+ \\
& \left.2 \int_{0}^{\pi} L\left(y_{P}, y_{q}, x_{P}, x_{q}, \alpha\right) d \alpha\right|_{x_{P} \neq x_{q}, y_{P} \neq y}
\end{aligned}
$$

so with all integrals evaluated numerically the problems with the singularities have been removed. Considering now the last two terms in equation (14) and denoting these by $R_{2}$ say then

$$
\begin{aligned}
& R_{2}=\left(H\left(A_{C}, b, \beta_{q}\right)-J\left(b, \beta_{q}\right) y_{q}^{2}\right) I^{(3)} \\
& =2\left\{\int_{0}^{\pi} e^{-i \sqrt{A_{C}} R}\left[\frac{H-y_{q}^{2}}{R}\right] L d \alpha\right\} \\
& =2 \int_{0}^{\pi} e^{-i \sqrt{A_{C}} R} \Xi\left(H, J, y_{q}\right) d \alpha, \text { say } \\
& =2 \int_{0}^{\pi}\left(e^{-i \sqrt{A_{C}} R}-1\right) \Xi\left(H, J, y_{q}\right) d \alpha+2 \int_{0}^{\pi} \Xi\left(H, J, y_{q}\right) d \alpha \\
& =2 \Re e\left\{\int_{0}^{\pi}\left(1-i \sqrt{A_{C}} R-\frac{A_{C} R^{2}}{2 !}+\ldots . .-1\right) \Xi d \alpha\right\} \\
& +2 \int_{0}^{\pi} \Xi\left(H, J, y_{q}\right) d \alpha \\
& =A_{C} \int_{0}^{\pi} R\left(H-J y_{q}^{2}\right) d \alpha+\left.2 \int_{0}^{\pi} \Xi\left(H, J, y_{q}\right) d \alpha\right|_{x_{P} \neq x_{q}, y_{P} \neq y}
\end{aligned}
$$

so that when $x_{P}=x_{q}, y_{P}=y_{q}, \alpha=0$ the first term vanishes so that

$$
\begin{aligned}
& R_{2}=A_{C} \int_{0}^{\pi} R\left(H-J y_{q}^{2}\right) d \alpha+\left.2 \int_{0}^{\pi} \Xi\left(H, J, y_{q}\right) d \alpha\right|_{x_{P} \neq x_{q}, y_{P} \neq y} \\
& +\frac{1}{2}\left[\log _{e}\left|\tan \frac{\alpha}{4}\right|\right]_{-\pi}^{\pi}
\end{aligned}
$$

i.e.

$$
R_{2}=A_{C} \int_{0}^{\pi} R\left(H-J y_{q}^{2}\right) d \alpha+\left.2 \int_{0}^{\pi} \Xi\left(H, J, y_{q}\right) d \alpha\right|_{x_{P} \neq x_{q}, y_{P} \neq y}
$$
finally for the term $y_{q}\left(x_{q}-x_{P}\right) I^{(1)}$, special attention is required only when the point $\mathrm{P}$ is on the discs $S_{1}$ or $S_{2}$, or in one of the four corners. Using a Plemelj formulae type analysis or y indenting the contour it may be shown that on the discs $S_{1}$ or $S_{2}$ the limiting value of this expression is $2 \pi$ and for the corners it is $\pi$.

\section{CONCLUSIONS}

An integral formula has been derived that allows for the computation of the velocity in a swan neck duck that allows for the possibility of non-cylindrical flow. The case when the integrands become singular have been examined so that all singular terms are catered for. This paper along with the accompanying paper by Pavlika[1] when coded and verified should make a significance advance in non-cylindrical flow bounded by two coaxial cylinders of varying radii. The matrix formulation uses the trapezoidal rule to perform the numerical integration but other techniques such as Simpson's rule or quadrature techniques may be required.

\section{ACKNOWLEDGMENT}

The author would like to thank his former Ph.D supervisor the late Dr Derek Payne, Director of Oakbark Consultants Ltd and Dr Jane Cousins a former Research Student of Dr Payne for their contribution to the development of this material.

\section{REFERENCES}

[1] V. Pavlika. "Vector Field Methods and the Hydrodynamic Design of Annular Ducts," Ph.D thesis, University of North London, Chapter X, pp. 246-260, 1995.

[2] G. I. Taylor. "Experiments on the motion of solid bodies in rotating fluids," in Proc. the Royal Society A 1104, vol. 213, no. 18, pp. 2213.

[3] G. B. Arfken and H. J. Weber, Mathematical Methods for Physicists, $5^{\text {th }}$ Edition, Academic Press, 1966.

[4] G. F. Roach, Green's Functions, $2^{\text {nd }}$ Edition, Cambridge University press, pp. 52-58, 1982.

[5] J. B. Diaz, "On Cauchy's problem and fundamental solutions," Contributions to the theory of partial differential equations, Princeton University Press, 1954, pp. 110-115.

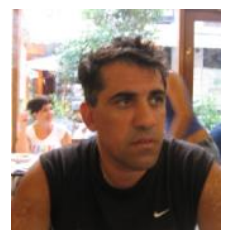

Vasos Pavlika has a B.Sc (Hons) in Mathematics and Physics (1987), an M.Sc in Mathematics (1988), a Ph.D in Applied Mathematics (1995) and a PGCE in teaching Mathematics and Physics in Post-Compulsory Education (1999). He has worked at the University of North London (1988), Newham Sixth Form College (1999), Havering College of Further and Higher Education (2000), The University of East London (2000), The London School of Economics and Political Science (2002-present), The University of Gloucestershire (2002) and The University of Westminster (2003-2010), SOAS, University of London (2003-Present), The University of Oxford (2004-Present), St George's, University of London (2011-2013). Dr Pavlika is a member of the WSEAS, an Editor of the Mathematical Journal of the Antarctica and a member of the WCNA. He has a certificate of Merit for a paper presented at the International MultiConference of Engineers and Computer Scientists 2007, Hong-Kong 21-23 March 2007 and Winner of the Best Paper Award for a paper presented at the International MultiConference of Engineers and Computer Scientists 2007, San Francisco 24-26 October 2007 and has over 20 publications including chapters in the following books: 
The Calculation of Annular Duct Geometries by Prescribing a Velocity Distribution on the Pressure Surface and the Radius of the Suction Surface", pp2375-2383, Lecture Notes in Engineering and Computer Science, Newswood Limited 2007, ISBN 978-988-98671-4-0, Magnetostatic field calculations associated with thick Solenoids in the Presence of Iron using a Power Series expansion and the Complete Elliptic Integrals" Applied Numerical Methods, Springer Verlag 2007 and The calculation of axisymmetric duct geometries for incompressible rotational flow with blockage effects and body forces". Advances in Computational Algorithms and Data Analysis, Springer Verlag 2008. His research Interests include: teaching in $\mathrm{HE}$, theoretical and computational fluid dynamics and
Magnetostatics. He is a Fellow of the Institute of Mathematics and its Applications (FIMA, CMath, CSci and CMathTeach), a Fellow of the Institution of Analysts and Programmers (FIAP), a Member of the Institute of Physics (MInstP, CPhys), a Fellow of the British Computer Society (FBCS CITP and CEng), a Member of the Institution of Electrical Engineers (MIEE and MIET) and a Member of the British Society for the History of Mathematics. 